\title{
LEI DE INOVAÇÃO TECNOLÓGICA: INSTRUMENTO EFETIVO DE INCENTIVO A INOVAÇÃO E A PESQUISA NO BRASIL?
}

\section{LAW OF TECHNOLOGICAL INNOVATION: EFFECTIVE INSTRUMENT OF INCENTIVE FOR THE INNOVATION AND THE RESEARCH IN BRAZIL?}

\author{
José Matias Pereira ${ }^{1}$; Isak Kruglianska ${ }^{2}$ \\ ${ }^{1}$ Universidade de Brasília - UNB - matias@unb.br \\ ${ }^{2}$ Universidade de São Paulo - USP - $\underline{\text { ikruglia@usp.br }}$
}

\begin{abstract}
Resumo
O objetivo deste artigo é aprofundar a discussão sobre as politicas de gestão da inovação no Brasil, a partir da avaliação da consistência da Lei de Inovação Tecnológica - LIT (Lei Federal nº. 10.973, de 02.12.2004) e do Decreto de regulamentação (Decreto $n^{\circ}$. 5563, de 13.10.2005) -. Estas normas legais estabelecem medidas de incentivo à inovação e à pesquisa científica e tecnológica no ambiente produtivo, com vistas à capacitação e ao alcance da autonomia tecnológica e ao desenvolvimento industrial do país -, através da análise das principais medidas explicitadas no seu texto. Utilizamos como referência nessa avaliação as experiências de alguns países bem sucedidos na implementação de políticas industrial e tecnológica. Ficou evidenciado nesse esforço que, a aprovação e a regulamentação da lei de inovação ainda apresentam sérias deficiências como instrumentos de suporte ao desenvolvimento tecnológico do Brasil.
\end{abstract}

Palavras-chave: Lei de inovação, política industrial e tecnológica, crescimento econômico, desenvolvimento, Brasil.

\section{Introdução}

Observa-se que o tema abordado neste artigo é significativamente amplo, complexo e polêmico, visto que existe uma parcela da comunidade científica que discordam da necessidade de que se tenha uma política de inovação tecnológica. A base de argumentação desse segmento da comunidade científica é o de que deve prevalecer sempre a ampla liberdade do pesquisador de determinar o objeto e escopo de suas pesquisas, ao passo que ao poder público fica o papel de financiamento das atividades de pesquisa. Aceitamos, neste artigo, que é relevante e necessário a existência de uma política nessa área. 
No debate sobre a construção de uma política industrial e tecnológica e de uma lei de inovação torna-se relevante fazer uma análise do sistema de Ciência e Tecnologia do Brasil (SCTB). Assim, iremos adotar neste artigo, de maneira preferencial, uma abordagem funcionalista, sistêmica em que as organizações e relações foram, inicialmente, consideradas como instituições "tipo-ideal" weberiano, para facilitar a identificação e estudo dos elementos que formam este complexo sistema. Vamos levar em consideração também o conceito de "racionalidade limitada" de Herbert Simon (1959, 1965, 1978). Assim, partimos do pressuposto que, o SCTB, apesar de apoiar-se numa base estrutural razoável, necessita ser fortalecida de várias maneiras para que possa ser ampliada, consolidada e garantida contra eventuais instabilidades no futuro.

Sustenta Morgan (1980) que a abordagem funcionalista é essencialmente ortodoxa e que ela é "baseada na pressuposição de que a sociedade tem uma existência concreta e real, e um caráter sistêmico orientado para produzir um estado de coisas que sejam ordenadas e reguladas". Ao estabelecer a distinção entre estabilidade e mudança como aspectos específicos da ordem e do conflito, respectivamente, Dahrendorf (1959) alerta que não tem a intenção de afirmar que a teoria da ordem assume que a sociedade seja estática (BURREL \& MORGAN, 1979). Sua preocupação é mostrar como as teorias funcionais são essencialmente correlacionadas com os processos que servem para manter os padrões de um sistema como um todo. Nesse sentido, as teorias funcionalistas são consideradas como estáticas no sentido de que elas procuram explicar o status quo. Aguiar (1991), utilizando o método de análise funcional, para descrever a ambiência de ciência e tecnologia no Brasil, busca explicar como os elementos que compõem o sistema nacional procuram se estabilizar, integrar e atuar harmonicamente.

\section{Motivação para a construção de um modelo científico e tecnológico}

A acirrada disputa pela apropriação da informação, do conhecimento e do desenvolvimento da inovação no mundo contemporâneo, decorrente do processo de globalização indica a necessidade do Brasil de construir um modelo de desenvolvimento tecnológico autônomo. Isso torna explícito que a Lei de Inovação Tecnológica - cujo teor deve refletir claramente que a geração de conhecimento e a formação de recursos humanos são funções da universidade, e que a inovação tecnológica ocorre no âmbito das empresas -, se apresenta como um instrumento relevante para reduzir a dependência tecnológica do país. 
A inovação tecnológica compreende a introdução de produtos ou processos tecnologicamente novos e melhorias significativas que tenham sido implementadas em produtos e processos existentes. Considera-se uma inovação tecnológica de produto ou processo aquela que tenha sido implementada e introduzida no mercado - inovação de produto - ou utilizada no processo de produção - inovação de processo- (OCDE, Manual Oslo, p. 35).

A partir dessas observações, formula-se a seguinte pergunta: $\mathrm{O}$ arcabouço legal de estímulo à inovação se apresenta como um instrumento efetivo de apoio à inovação e a pesquisa científica e tecnológica no Brasil?

Para responder esta pergunta partimos do pressuposto que o Brasil ainda não construiu um arcabouço institucional na área científico-tecnológica suficientemente consistente capaz de definir adequadamente quais são as atividades de pesquisa de real interesse tecnológico para o país. Assim, temos como propósito debater neste artigo, a partir das experiências de alguns países bem sucedidos na implementação de políticas industrial e tecnológica, se a construção de um moderno arcabouço institucional na área de desenvolvimento científico e tecnológico, no qual deve estar incluso a questão da inovação, é essencial para o crescimento econômico do Brasil. A investigação - que é explicativa quanto aos fins e bibliográfica quanto aos meios - está apoiada em vários estudos e documentos, como por exemplo, Dahlman \& Frischtak, 1990; Pavitt, 1991, 1998; Coutinho e Ferraz, 1994; Matias-Pereira e Kruglianskas (2004 e 2005), na Lei de Inovação Tecnológica (Lei Federal no 10.973/2004) e no Decreto de regulamentação (Decreto $n^{\circ}$. 5563, de 13.10.2005).

\section{Análise das Experiências de Países de Sucesso na Promoção de CT\&I}

A abordagem da experiência em CT\&I de alguns países bem sucedidos nessas áreas é oportuna para a compreensão do propósito deste estudo. Argumenta-se que, tanto o Parlamento como a sociedade organizada brasileira, na discussão para promover o aperfeiçoamento do projeto de lei de inovação brasileira, precisa levar em consideração as estratégias que esses países adotaram em C\&T para fomentar o processo de desenvolvimento econômico e social.

$\mathrm{Na}$ maior parte dos países integrantes da Organização de Cooperação e Desenvolvimento Econômico (OCDE, 1996 e 1997b), os governos estão adotando políticas para contrapor os efeitos provocados pelo nível elevado de abertura ao exterior que se seguiu à importante redução de barreiras tarifárias que foram implementadas. As políticas de competitividade adotadas nos países da OCDE 
(1996), visando o aumento das exportações estão orientadas de forma seletiva. Tem havido uma diminuição dos subsídios diretos às empresas e ênfase no apoio ao investimento em setores intensivos em tecnologia e à constituição de infra-estrutura necessária para alcançar objetivos de crescimento de longo prazo. As pequenas e médias empresas e a organização de clusters industriais recebem tratamento diferenciado na alocação dos investimentos. Naqueles países a política industrial é percebida de uma maneira mais ampla e sua função é articular e envolver empresas de diferentes setores e atividades, tendo a tecnologias de informação e as comunicações como molas impulsoras do processo (LASTRES e ALBAGLI, 1999; e, LORA, 2001).

Apoiado na experiência dos países industrializados se argumenta que o caminho para o desenvolvimento econômico e social passa, necessariamente, pela eficiência tecnológica (MATESCO, 2001). Essa realidade é reforçada, também, pelo sucesso de alguns países emergentes que estão gerando riqueza a partir do conhecimento alcançado em centros de pesquisa (MALDONADO, 1998). A partir da experiência internacional, destacam-se as experiências da Coréia do Sul, França, Estados Unidos e Japão, cujas políticas industriais e tecnológicas refletem e influenciam os debates sobre o tema no Brasil (MATIAS-PEREIRA e KRUGLIANSKAS, 2005).

A experiência internacional referente às legislações que definem medidas para a promoção da ciência, tecnologia e inovação são reveladoras, apoiado nos exemplos acima assinalados, servem como ilustração, para comprovar que os países que consideram C\&T como atividade estratégica constrói sistemas legais que estimulam, de forma coordenada, o desenvolvimento científico, tecnológico e a inovação no setor industrial. Sem desconsiderar a dimensão física, a diversidade regional e a complexidade econômica, política, cultural e populacional dos países citados, pode-se argumentar que a consecução das metas na área de ciência e tecnologia passa pela consolidação de um arcabouço legal, cujo conteúdo permita dinamizar a relação entre universidades, institutos de pesquisa e o setor produtivo nacional.

\section{A Conexão entre as Leis sobre Inovação e Pesquisa da França e do Brasil}

A partir das considerações anteriores torna-se relevante analisar a relação e os aspectos mais relevantes da Lei de Inovação e Pesquisa da França - Lei no 99-587 -, e da Lei de Inovação Tecnológica do Brasil - Lei Federal nº 10.973/04 -.

A Lei sobre Inovação e Pesquisa da França, no 99-587, de 12 de julho de 1999, estabelece os procedimentos legais da relação público-privada, além de criar mecanismos que estimulem a inovação 
tecnológica no ambiente universitário. Tem como objetivo facilitar a transferência de pesquisa financiada pelo setor público para a indústria e a criação de empresas inovadoras. A experiência mostra que a utilização econômica dos resultados da pesquisa é um fator básico do dinamismo da economia, o número de empresas criadas a cada ano utilizando os resultados de pesquisa financiada pelo setor público permanece demasiado baixo. São, entretanto, essas empresas que tem o mais forte potencial para crescimento. Observa-se que, a citada Lei de inovação e pesquisa tem como propósito reverter esta tendência e proporcionar um contexto legal que fomente a criação de empresas inovadoras de tecnologia, sobretudo por parte de pessoas jovens, sejam eles pesquisadores, estudantes ou empregados.

Observa-se que, a Lei francesa está estruturada em quatro seções, que cuidam da: mobilidade dos pesquisadores em direção à indústria e as empresas; a cooperação entre estabelecimento de pesquisa do setor público e as empresas; o quadro geral fiscal para empresas inovadoras; e o quadro geral jurídico para empresas inovadoras. A comparação do conteúdo da Lei francesa indica que a mesma influenciou decisivamente o projeto de lei de inovação tecnológica do Brasil. A similitude entre o teor da Lei sobre Inovação e Pesquisa da França e o texto do projeto de lei nacional é bastante evidente.

Por sua vez, a Lei de inovação tecnológica brasileira está orientada para a criação de ambiente propício a parcerias estratégicas entre as universidades, institutos tecnológicos e empresas; o estímulo à participação de instituições de ciência e tecnologia no processo de inovação; e o incentivo à inovação na empresa. Possibilita autorizações para a incubação de empresas no espaço público e a possibilidade de compartilhamento de infra-estrutura, equipamentos e recursos humanos, públicos e privados, para o desenvolvimento tecnológico e a geração de processos e produtos inovadores.

Define, ainda, regras para que o pesquisador público possa desenvolver pesquisas aplicadas e incrementos tecnológicos. Para isso criou mecanismos como: a bolsa de estímulo à inovação e o pagamento ao servidor público de adicional variável não-incorporável à remuneração permanente, ambos com recursos captados pela própria atividade; a participação nas receitas auferidas pela instituição de origem com o uso da propriedade intelectual e a licença não-remunerada para a constituição de empresa de base tecnológica. Autorizam também o aporte de recursos orçamentários diretamente à empresa, no âmbito de um projeto de inovação, sendo obrigatórias a contrapartida e a avaliação dos resultados. São ainda instrumentos da Lei a encomenda tecnológica, a participação estatal em sociedade de propósito específico, e os fundos de investimentos. Para o governo federal, a 
Lei ao estimular a inovação no setor produtivo, irá incentivar a emancipação tecnológica do País e mudar o quadro atual onde $73 \%$ dos cientistas estão atuando nas instituições públicas e apenas $11 \%$ nas empresas privadas.

\section{As normas definidoras da Lei de inovação tecnológica brasileira}

O Brasil - com a entrada em vigor da Lei de Inovação Tecnológica (LIT), no início de dezembro de 2004 - passou a contar com um novo instrumento de fomento à inovação e à pesquisa científica e tecnológica no ambiente produtivo, com vistas à capacitação e ao alcance da autonomia tecnológica e ao desenvolvimento industrial do país. As definições conceituais que levam à compreensão das disposições normativas da LIT estão contidas no capítulo I do texto. O Capítulo II cuida do estímulo à construção de um ambiente de inovação, trazendo os comandos permissivos para a interação profícua entre as entidades de pesquisa e a iniciativa privada. Destaca-se, em especial, a sinergia para viabilização de empresas emergentes, por meio do processo de incubação, e a colaboração com empresas consolidadas. O capítulo III está orientado para a geração de estímulos para a participação das entidades públicas de pesquisa no processo de inovação. Preocupa-se em definir normas que permitam a transferência e o licenciamento de tecnologia das universidades e institutos de pesquisa públicos do país para o setor produtivo nacional.

A Lei de Inovação busca promover e incentivar o desenvolvimento científico, a pesquisa e a capacitação tecnológicas de forma a viabilizar o desenvolvimento, conforme define os artigos 218 e 219 da Constituição Federal. O estímulo à inovação coloca no mercado, produtos e serviços mais competitivos, gera emprego, renda e desenvolvimento. E uma das maneiras de ampliar esta gama de benefícios está nas incubadoras de empresas. Trata-se de uma ambiente que proporciona a possibilidade de compartilhamento de infra-estrutura, equipamentos e recursos humanos, públicos e privados às empresas nascentes. Neste aspecto, a nova legislação pretende regularizar a situação das empresas privadas dentro das universidades e criar um sistema de incentivos fiscais para seu desenvolvimento, a começar pela contratação de pesquisadores e empresas sem licitação. Ao colocar a inovação como foco principal, a nova Lei de Inovação reconhece que não basta para um país fazer tão só Ciência e Tecnologia e Pesquisa e Desenvolvimento. É preciso transformar isto em novos produtos e processos ou melhorar o que já existe, para que os produtos finais sejam desejados pelos mercados nacional e internacional. 
Observa-se que a Lei de Inovação Tecnológica está organizada em torno de três eixos: a constituição de ambiente propício a parcerias estratégicas entre as universidades, institutos tecnológicos e empresas; o estímulo à participação de instituições de ciência e tecnologia no processo de inovação; e o incentivo à inovação na empresa. Ela prevê autorizações para a incubação de empresas no espaço público e a possibilidade de compartilhamento de infra-estrutura, equipamentos e recursos humanos, públicos e privados, para o desenvolvimento tecnológico e a geração de processos e produtos inovadores. Também estabelece regras para que o pesquisador público possa desenvolver pesquisas aplicadas e incrementos tecnológicos. Seus principais mecanismos são: bolsa de estímulo à inovação e o pagamento ao servidor público de adicional variável não-incorporável à remuneração permanente, ambos com recursos captados pela própria atividade; a participação nas receitas auferidas pela instituição de origem com o uso da propriedade intelectual e a licença não-remunerada para a constituição de empresa de base tecnológica. A Lei também autoriza o aporte de recursos orçamentários diretamente à empresa, no âmbito de um projeto de inovação, sendo obrigatórias a contrapartida e a avaliação dos resultados. São ainda instrumentos da Lei a encomenda tecnológica, a participação estatal em sociedade de propósito específico, e os fundos de investimentos.

Para potencializar os instrumentos da Lei, o governo federal criou a Agência Brasileira de Desenvolvimento Industrial (ABDI). A Agência, instalada no início deste mês, será responsável pela execução e acompanhamento da Política Industrial, Tecnológica e de Comércio Exterior do governo. A ABDI tem como função articular ações e estratégias da política industrial por meio do apoio ao desenvolvimento do processo de inovação e do fomento à competitividade do setor produtivo. A Agência é composta por órgãos do Poder Executivo (ministérios do Desenvolvimento, Indústria e Comércio Exterior, da Ciência e Tecnologia e Casa Civil, por exemplo) da sociedade civil (Sebrae, Apex-Brasil e Confederação Nacional da Indústria) e da iniciativa privada. A previsão é que o novo órgão esteja em pleno funcionamento dentro de um mês. Além disso, o Ministério da Ciência e Tecnologia (MCT) já está trabalhando num projeto de lei criando regime fiscal favorável à inovação.

\section{Principais características e deficiências da lei de inovação}

Tendo como objetivo identificar as principais características e fragilidades da lei de inovação tecnológica do Brasil, realizamos um total de dezenove entrevistas abertas, com atores dos principais segmentos envolvidos no debate da construção da Lei de Inovação. A partir dessas entrevistas 
podemos argumentar que as principais características e deficiências da Lei de Inovação são as seguintes:

a) A criação de mecanismos jurídicos e financeiros para as empresas, universidades e cientistas interagirem, na procura por soluções inovadoras, foi aceito pela maioria dos entrevistados como uma medida necessária, visto que a parceria até então existente ocorria de forma precária e com um nível baixo de transparência. Esperam, a partir da lei e de sua regulamentação, que se abram novas possibilidades para as partes constituir fundos, criar sociedades anônimas, tomar empréstimos, receber os incentivos e dividir as patentes.

b) Para uma parcela significativa dos cientistas entrevistados a lei é positiva, conceitualmente falando, por reconhecer a empresa como o local da inovação. Para esse segmento existe uma sinalização clara de que o governo federal está orientando medidas para a geração de estímulos à inovação, flexibilizando atividades e relações das instituições científicas e tecnológicas. Para eles a lei busca enfrentar o desafio de criar no Brasil as condições necessárias para que o país possa dar um salto consistente no campo tecnológico, em particular quando reconhece que entre as soluções apontadas para corrigir rumos está incrementar a interação entre Instituições Científicas e Tecnológicas incluindo os Centros de Educação Tecnológica -, e o parque produtivo.

c) Entre as medidas necessárias para o desenvolvimento das inovações no país, em particular as medidas defendidas pelos empresários, destacam-se as que sustentam que as despesas de P\&D sejam deduzidas no lucro tributável; desoneração dos investimentos de $\mathrm{P} \& \mathrm{D}$; definição de incentivos diretos para a formação dos empresários em know how empresarial, estratégias de marketing, diferenciação competitiva e princípios de $\mathrm{P} \& \mathrm{D}$; oferta de incentivos diretos para a formação da infra-estrutura de $\mathrm{P} \& \mathrm{D}$; financiamentos privilegiados de pesquisa para projetos envolvendo universidade-indústria; apoio financeiro e técnico-jurídico para patenteamento de novas descobertas realizadas no Brasil e no exterior.

d) Todos os juristas entrevistados sustentam que existem imperfeições e carências no teor da lei de inovação, como por exemplo, a sua amplitude e excesso de detalhes, a falta de definições claras para a geração de estímulos e apoio à micro e pequenas empresas do país. Destacam, também, as falhas na forma de definição das normas que tratam da interação entre os três atores principais para o sucesso da lei de inovação, ou seja, o inventor, a universidade e os institutos de pesquisa, e o capital de risco. Para eles a LIT não definiu, também, as regras dessa relação em contrato, que ainda permanecem obscuras. Estas constatações reforçam a argumentação dos entrevistados de que a implementação das medidas 
definidas na lei inovação irá exigir o envolvimento de toda sociedade organizada na sua regulamentação, para que possa sanar esses defeitos e vir a cumprir os seus objetivos, em particular na geração dos estímulos adequados à inovação, flexibilizando atividades e as relações das instituições científicas e tecnológicas.

e) A argumentação utilizada pelo governo federal - tendo como base à legislação existente nos países mais avançados - para permitir o afastamento de pesquisadores empreendedores, para que possam desenvolver atividade empresarial relativa à produção de bens diretamente decorrentes de criação de sua autoria, que irá permitir transformar o conhecimento em bens tangíveis para a sociedade é aceita pela maioria dos entrevistados. Ressaltam os entrevistados - professores-pesquisadores de universidades públicas e centros de pesquisa - sobre a necessidade de se analisar com maior profundidade os riscos de um esvaziamento das universidades públicas, considerando que no texto da proposta não se prevê mecanismos que permitam às universidades controlar adequadamente o trabalho dos pesquisadores que se afastarem para desenvolver projetos de pesquisa no setor privado. Nesse sentido, defendem que a regulamentação da lei de inovação deve preocupar-se principalmente com a transparência na relação entre os professores das universidades e empresas.

f) $\mathrm{Na}$ parte que cuida da gestão da inovação, existe uma percepção por parte de uma parcela dos professores - pesquisadores que a lei é carente de normas definidoras que permitam às universidades controlar melhor o trabalho dos pesquisadores que venham a se afastar do ambiente acadêmico para desenvolver projetos no setor privado. Para esses entrevistados é indesejável para a sociedade que o interesse do pesquisador prevaleça em relação ao da instituição a que ele está ligado. Nesse sentido argumentam que é preciso preservar os interesses das instituições de pesquisa para impor limites à iniciativa privada. Observa-se que, uma parcela dos professores que atuam como consultores ou que executam projetos para o setor privado usa as fundações de apoio das universidades, que firmam contratos e movimentam recursos praticamente sem prestar contas às universidades. Caso isso não seja corrigido na regulamentação da lei de inovação, corre-se o risco do interesse do pesquisador acabar prevalecendo em relação ao da instituição a que ele está ligado.

Sob esta ótica, a lei não está sendo aceita pacificamente, por uma parcela de professores/pesquisadores e dirigentes de associações de docentes, como uma solução para os problemas de desenvolvimento tecnológico brasileiro. Para esse segmento, a lei representa mais um estágio no processo de aprofundamento da privatização do setor público. Argumentam que o papel das universidades públicas não é o de fornecer mão-de-obra e capacidade instalada a custo zero para o setor privado, nem pagar a 
conta da falta de investimento estratégico em tecnologia. Seu papel é estabelecer um ambiente onde a pesquisa - não adaptação tecnológica -, ensino crítico e de qualidade, bem como extensão, são exercidos de modo indissociável, propiciando bases sólidas para o desenvolvimento social, cultural e tecnológico do país.

g) A maioria dos entrevistados - em particular os empresários e pesquisadores - entendem que o processo de estruturação de competências tecnológicas e organizacionais, no interior das firmas requer um amplo e constante acesso às fontes de informação básica, visando transformar informações em conhecimento. Para que isso ocorra, as empresas - cujo propósito é desenvolver seus próprios sistemas de aprendizado tecnológico e organizacional - precisam criar departamentos específicos de P\&D (ROUSSEL, SAAD \& BOHLIN, 1992). Assim, argumentam que a lei de inovação não levou em consideração a essencialidade dessas questões, visto que sem tecnologia as possibilidades de competir nos mercados mundiais ficam comprometidas. Nesse sentido, sustentam que a criação e manutenção dos centros de $\mathrm{P} \& \mathrm{D}$ e a conseqüente valorização dos recursos humanos altamente qualificados que atuam nesses centros deve ser uma medida prioritária para o país. Esse esforço irá contribuir para reduzir o papel das empresas multinacionais como principais produtoras e detentoras de tecnologia de ponta. A geração de estímulos para elevação na prioridade dada pelas empresas para a área de ciência e a tecnologia tem como propósito evitar que continue ocorrendo o processo de desmantelamento de centros de $\mathrm{P} \& \mathrm{D}$ e a conseqüente dispensa de recursos humanos de alto nível.

h) A Lei de Inovação, para uma parcela dos entrevistados, irá contribuir para consolidar a relação produção, pesquisa e União, visto que busca desenvolver esforços de diversos ministérios e da sociedade. Entendemos que a relação com o setor privado não deve enfraquecer as universidades, porque sem elas não haverá bens de conhecimento e sem bens de conhecimento não há transferência, nem inovação e nem produção. Por sua vez, diversos aspectos e desafios precisam ser vencidos como a integração da Lei de Inovação à nova Política Industrial e sua ação coordenada às políticas estaduais e municipais.

i) Todos os entrevistados concordaram que a LIT somente produzirá resultados concretos se as indústrias necessitarem inovar. Lembram, entretanto, que o processo de inovação não ocorre pela oferta de mecanismos, mas quando é gerada a necessidade de inovar. A demanda P\&D por parte da empresa decorre da sua necessidade de competir em novos mercados, com níveis de exigências mais sofisticados, visto que as suas vendas não estarão ocorrendo em função do preço, mas pela qualidade 
do produto e do segmento do mercado. Dessa forma, a LIT apenas abre possibilidades, mas não garante que as agências de governo incentivem adequadamente o uso dos instrumentos disponíveis.

j) Os representantes do setor privado entendem que a decisão das empresas transnacionais ou globais de implantar novos centros de tecnologia, ou seja, liderar do país, com pesquisa nacional, o desenvolvimento de novos produtos depende de diversos fatores. $\mathrm{Na}$ maioria dessas empresas não existe, necessariamente, uma matriz concentrando todas as relações de P\&D. Assim, para cada segmento de negócios se podem ter várias competências, em países diferentes. Dessa forma

argumentam que a cooperação entre empresa e academia não basta. É necessário existir um arcabouço institucional consistente, a partir de uma política industrial e tecnológica consistente, que permita que os dirigentes dessas empresas globais possam confiar a brasileiros a gestão de um centro de tecnologia. No Brasil isso já vem ocorrendo com a política de fomento na área de informática, por meio da Lei de Informática, que viabilizou a produção local, exigindo uma contrapartida de investimento das empresas em P\&D. Diversas empresas, como por exemplo, a Motorola, a HP e a Siemens se beneficiaram dessa política, e dessa forma criaram mais empregos e firmaram parcerias com universidades.

\section{Conclusões}

É perceptível que a regulamentação da Lei de Inovação - que foi organizada com os objetivos de criar um ambiente propício a parcerias estratégicas entre as universidades, institutos tecnológicos e empresas; incentivar à participação de institutos de ciência e tecnologia no processo de inovação; e, estimular à inovação na empresa - busca potencializar a aplicação de recursos em pesquisa e desenvolvimento, tanto nas instituições públicas como nas empresas, contribuindo para aumentar o desenvolvimento e a competitividade dos produtos brasileiros. Possibilita ainda, que os inventos de criadores independentes sejam adotados pelas instituições científicas e tecnológicas para futuro desenvolvimento, incubação e industrialização pelo setor produtivo, além de prever a participação da União em iniciativas de base tecnológica junto com empreendedores, ao mesmo tempo em que definem incentivos fiscais para as empresas que fazem inovação. Torna, também, mais dinâmica as relações de trabalho das instituições científicas e tecnológicas, além de estabelecer o regime de comercialização das inovações geradas nessas instituições e, em especial, cria os mecanismos necessários que favoreçam o ambiente de criação e inovação dentro das empresas. 
A aprovação e a regulamentação da Lei de Inovação Tecnológica (LIT) apresentam-se como uma medida necessária para permitir que o país passe a dispor de mais um instrumento de suporte para a criação de ambiente propício a parcerias estratégicas entre as universidades, institutos tecnológicos e empresas; o estímulo à participação de instituições de ciência e tecnologia no processo de inovação; e o incentivo à inovação na empresa. Pode-se observar, ainda, que a lei de inovação representa o fechamento de um ciclo, que partiu da discussão focada nos recursos e atingiu os aspectos institucionais.

Inúmeras questões complexas não foram tratadas adequadamente na regulamentação da Lei, como por exemplo, a excessiva rigidez que prevalece na gestão de recursos humanos, financeiros e materiais nas instituições de pesquisa, bem como a necessidade de harmonização de seus dispositivos com os demais diplomas legais que regulam a matéria no país. Dentre essas deficiências podemos destacar, por exemplo, as duas principais medidas contidas na LIT para estimular as empresas investirem em inovação: a concessão de subvenção econômica e a utilização de benefícios de incentivos fiscais pelas empresas ainda não se concretizaram.

A criação de uma subvenção econômica pela LIT tem como objetivo permitir ao governo financiar projetos da iniciativa privada diretamente, com recursos públicos. Verifica-se, na prática, que esses benefícios - a fundo perdido - ainda não estão sendo utilizados pelas empresas porque persistem inúmeras dúvidas jurídicas para a sua execução. Por sua vez, a reforma promovida nos incentivos fiscais existentes na legislação brasileira - via renúncia fiscal - para reduzir os custos de atividades de pesquisa e desenvolvimento de novos produtos na empresas, ainda não foram definidos de forma clara pela Secretaria da Receita Federal. Dessa forma, a falta de clareza das normas sobre dedução de despesas pelas empresas e em que situações a contratação de pesquisadores gerará benefícios fiscais para as empresas são fatores inibidores da utilização desses incentivos fiscais.

Ressaltamos, assim, que apesar de ser um diploma legal que ainda possui deficiências, a lei de inovação tecnológica representa um ponto de partida importante para fomentar a construção de um modelo de desenvolvimento tecnológico autônomo no Brasil. O texto da LIT e do decreto de sua regulamentação evidencia os limites do possível, num país onde a política de Estado com relação à inovação é significativamente frágil. Isso nos permite argumentar que as partes mais relevantes envolvidas nessa questão - parlamento, governo federal, empresas e universidades e centros de pesquisa -, devem continuar aprofundando o debate sobre o tema objeto deste estudo.As análises e os argumentos utilizados neste artigo permitiram responder, mesmo que parcialmente, a pergunta 
formulada no início deste artigo. Nela questionamos se "a lei de inovação - aceita como parte essencial do arcabouço institucional para fortalecer as áreas de pesquisa e da produção de conhecimento no Brasil -, poderá fomentar adequadamente a criação de novos ambientes, propícios à geração e absorção de inovações, atuando como ferramenta de apoio à Política Industrial e Tecnológica do Brasil". Verifica-se que nesta análise, ficaram algumas dúvidas relevantes sobre a consistência da lei de inovação e sua regulamentação, que entre outros aspectos, deve funcionar como instrumento de suporte para romper o ciclo vicioso da dependência tecnológica do país.

\begin{abstract}
In this article we have as objective to deepen the quarrel on the politics of management of the innovation in Brazil, from the evaluation of the consistency of the Law of Technological Innovation LIT (Federal Law $n^{\circ}$. 10.973/2004) and of the Decree of regulation (Decree $n^{\circ} .5563 / 2005$ ) -. These rules of law establish measured of incentive to the innovation and the scientific and technological research in the productive environment, with sights to the qualification and the reach of the technological autonomy and the development industrial of the country -, through the analysis of the main measures defined in its text. We use as reference in this evaluation the experiences of some successful countries in industrial and technological the implementation of politics. He was evidenced in this effort that, the approval and the regulation of the innovation law still present serious deficiencies as instruments of support to the technological development of Brazil.
\end{abstract}

Key words: Law of innovation, industrial and technological politics, economic growth, development, Brazil.

\title{
Referências
}

Abreu, M. e Verner, D. Long term Brazilian economic growth, Paris, OCDE, 1997.

Aguiar, A. C. Coordenação de uma rede nacional de informação em Ciência e Tecnologia: um plano prioritário do IBICT. Cien. Informação, v.9, n.1/2, 1981, p. 83-88.

Aguiar, A. C. Informação e atividades de desenvolvimento científico, tecnológico e Industrial . Cien. Informação, v. 20 , n. 1, p. 7-15, jan/jun. 1991.

Albuquerque, E. M. "Do catching up à fronteira tecnológica: notas sobre políticas industriais e a construção do sistema nacional de inovação no Japão" in Cassiolato, J. E. (coord.), Projeto de Pesquisa: Novas Políticas Industriais e Tecnológicas, Rio de Janeiro, Instituto de Economia da UFRJ, 1996.

Azevedo, Fernando (org.). As ciências no Brasil, 2 vols., Rio de Janeiro, RJ, Editora UFRJ, 1994.

Banco Central do Brasil. Indicadores Econômicos - 1998-2002, Brasília, DF, DEPEC/BCB, 2003.

Brasil. Congresso Nacional. Lei de Inovação Tecnológica (Lei no. 10.973/2004), Brasília, DF, Atos do Poder Legislativo, DOU, $n^{\circ} .232$, de 03.12.2004.

Brasil, Governo do Brasil. Decreto $\mathrm{n}^{\mathrm{o}}$. 5.563, de 13/10/2005. Regulamenta a Lei de Inovação Tecnológica. https://www.planalto.gov.br/ccivil_03/_Ato2004-2006/2005/Decreto/D5563.htm. Pesquisa feita em 14.10.2005. 
Brasil. Senado Federal. Constituição da República Federativa do Brasil de 1988, Brasília, DF, Senado Federal, 2005.

Brasil. Presidência da República. “Orientação Estratégica de Governo: Crescimento Sustentável, Emprego e Inclusão Social”, Brasília, DF, 2003. www.presidencia.gov.br. Pesquisa feita em 31.05.2006.

Brisolla, S. Indicadores quantitativos de ciência e tecnologia no Brasil, Campinas, SP, Núcleo de Política Científica e Tecnológica da Universidade de Campinas, 1993.

Burrel, G. e Morgan, G. Sociological Paradigms and Organizational Analysis -Elements of the Sociology of Corporate Life. London. Ed. Heinemann, 1979.

Caldas, Ruy C. "A construção de um modelo de arcabouço legal para a Ciência, Tecnologia e Inovação", em Parcerias Estratégicas. 11, 2001, p. 5-27.

Câmara dos Deputados. Projeto de Lei no. 7.282/2002, do Poder Executivo, Brasília, DF, CD, 2002.

Câmara dos Deputados. Projeto de Lei nº. 3.476/2004, do Poder Executivo, Brasília, DF, CD, 2004.

Coutinho, Luciano G. "Superação da fragilidade tecnológica e a ausência de cooperação", in Carlos Vogt e Eva Stal (orgs.), Ciência e tecnologia: Alicerces do desenvolvimento, Brasília, DF, CNPq, 1999, p. 107-124.

, e C. Ferraz (Orgs.). Estudo da competitividade da indústria brasileira, Campinas, São Paulo, Editora Unicamp/Papirus/MCT, 1994.

Cassiolato J. E. As novas políticas de competitividade: a experiência dos principais países da OCDE, T.D. 367, IE/IFRJ, julho 1996.

Cirillo, Walter. Caminhos para a inovação, Revista Uniemp, no 30, novembro, 2004, p. 18-20. www.uniemp.org.br. Pesquisa feita em 22.05.2006.

Cruz, C. H. B. "A universidade a empresa e a pesquisa que o país precisa", Revista Humanidades, 45, Editora Universidade de Brasília, 1999.

Cruz, C. H. B. Avaliação do projeto da lei de inovação: o lugar da inovação, Fórum da Lei de Inovação, Centro Minerva de Empreendedorismo, Escola Politécnica da Universidade de São Paulo, 05 de maio de 2004. Boletim Inovação Unicamp, 20.05.2004.

Dahlman, C. E Frischtak, C. (1990). "National systems supporting technical advance in industry”, Industry Series Paper, $\mathrm{n}^{\circ} 1.1990$, p. 1-32.

Dahrendorf, R. Class and Class Conflict in Industrial Society. Stanford, CA: Stanford University Press, 1959.

Erber, F. (2000). "O Padrão de desenvolvimento industrial e tecnológico e o futuro da indústria brasileira", Revista de Economia Contemporânea, Rio de Janeiro, RJ, IE-UFRJ, vol. 4, 2000, número especial.

Fleury, A. e Fleury, M.T.L. Aprendizagem e Inovação Organizacional. $2^{\mathrm{a}}$ ed. São Paulo: Atlas, 1997.

França. Lei sobre Inovação e Pesquisa Francesa, de 1999. www.education.gouv.fr/technologie. Pesquisa feita em 25.04.2006.

Freeman, C. The economics of technical change: critical survey. Cambridge Journal of Economics, v.18, 1984, p.463514.

Technology Policy and Economic Performance: Lessons from Japan, Pinter, 1987. "The National System of Innovation" in historical perspective. Cambridge Journal of Economics, v.19, n.1, 
Freeman, C. e Soete, L. (1994). The economics of industrial innovation. London: Pinter.

Gibbons, M., Limonges, C. et al. The new production of knowledge, London, Sage, 1994.

Guimarães, E. A. et al. A política científica e tecnológica, Rio de Janeiro, RJ, Jorge Zahar Editora, 1985.

Guimarães, R. Avaliação e fomento de C\&T no Brasil: Propostas para os anos 90, Brasília, DF, CNPq, 1995.

IBGE. Censo 2000, Rio de Janeiro, RJ, IBGE, 2002a. 194 págs.

IBGE. Indicadores econômicos, Rio de Janeiro, RJ, IBGE, 2003. www.ibge.gov.br

IBGE. Indicadores econômicos, Rio de Janeiro, RJ, IBGE. IEDI. (1998). Políticas industriais em países selecionados. São Paulo, Iedi, 2004.

INPI. Lei da Propriedade Industrial (Lei no 9.279), Rio de Janeiro, INPI, 1996.

Japan, Cabinet Decision, Science and Technology Basic Plan, http://www.cabinet.gov.jp

Lastres, Helena M. M. "Dilemas da política científica e tecnológica”, Ciência da Informação, v. 24, nº 2, 1995, p. 1-8.

Lastres, Helena M. M. e Albagli, S. (eds.). Informação e globalização na era do conhecimento, Rio de Janeiro, RJ, Campus, 1999.

Lastres, Helena M. M. e Cassiolato J. E. “A Política Tecnológica Japonesa”, Anais do Seminário sobre o Japão, Rio de Janeiro, 7-8 de dezembro 2000.

Levy-Strauss, C. Structural Anthropology. Trad. C.Jacobson \& B.C.Schoepf. New York: Basic Books. 1963, p.277

Lora, E. Competitividade: O motor do crescimento, Washington, DC, BID, 2001.

Maldonado, J. “Política Industrial no Japão”, Relatório NPI 06.1/98, Projeto de Pesquisa Apoiado pelo Instituto de Estudos para o Desenvolvimento Industrial - IEDI, Rio de Janeiro, RJ, IE/UFRJ, 1998.

March, J. G. e Simon, H. A. Teoria das Organizações. Rio de Janeiro: Ed. Fundação Getúlio Vargas, 1984.

Martino, J. Technological forecasting for decision making, 3rd ed., New York, McGraw-Hill, 1993.

Meis, L. e Leta, J. O Perfil da Ciência Brasileira, Rio de Janeiro, RJ, Editora UFRJ, 1996.

Matesco, V. (coord.). O atraso brasileiro na inovação tecnológica, Rio de Janeiro, RJ, IBRE/FGV, 2001.

Matias-Pereira, J. (2002a). Ciência e tecnologia e desenvolvimento no Brasil, Brasília, DF, Pesquisa Apoiada pelo Fundo de Pesquisa da Universidade de Brasília - PPGA /UnB. 2002a, 154 págs.

. "Science, Technology and Development in Brazil: The importance of creating a research culture in business and an entrepreneurial culture in the universities", Paper - Balas 2002 Annual Conference, The University of Tampa Press, 2002b, p. 1-33.

. "A Lei de Inovação Tecnológica como instrumento de apoio à construção de um modelo tecnológico autônomo no Brasil”, Paper - Altec 2003, México, 2003a, p. 1-15.

Economia Brasileira, $1^{\mathrm{a}}$ edição, Editora Atlas, São Paulo, 2003b. 154 págs.

- Avaliação do projeto da lei de inovação. Debatedor. Anais do Fórum da Lei de Inovação, Centro Minerva de

Empreendedorismo, Escola Politécnica da Universidade de São Paulo, 05 de maio 2004. 
- "The Importance of the Construction of Law of Brazilian Technological Innovation", Paper - Iberoamerican Conference 2003, São Paulo, 2003c, p. 1-18.

e Isak Kuglianskas. "Gestão de Políticas de Proteção à Propriedade Intelectual no Brasil”, Paper - XXIII Simpósio de Gestão de Inovação Tecnológica, NPTG/ANPAD, Curitiba, 2004, p. 1-15.

e Isak Kuglianskas. "Gestão de Inovação: A Lei de Inovação Tecnológica como Ferramenta de Apoio às Políticas

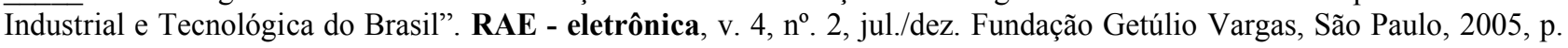
$1-21$.

Ministério do Desenvolvimento, Indústria e Comércio Exterior. Diretrizes de Política Industrial, Tecnológica e Comércio Exterior, Brasília, DF, MDICE, 2004. www.mdice.gov.br. Pesquisa feita em 25.05.2006.

Ministério do Planejamento, Orçamento e Gestão. Plano Plurianual - Avança Brasil - 2000/2003, Brasília, DF, PR/MPOG, 2000. 699 págs. $\overline{13.05 .2006 .}$

Plano Plurianual - 2004/2007, Brasília, DF, PR/MPOG, 2004. www.planejamento.gov.br. Pesquisa feita em

Ministério da Ciência e Tecnologia - MCT. Projeto Diretrizes Estratégicas para a Ciência, tecnologia e inovação em um horizonte de 10 anos, Brasília, DF, DECTI/MCT, 2000.

, MCT. Anais da Conferência Nacional de Ciência, Tecnologia e Inovação, Brasília, DF, CNPq/MCT, $2001 \mathrm{a}$.

, MCT. Livro Verde da Ciência, Tecnologia e Inovação, Brasília, DF, CNPq/MCT, 2001b.

, MCT. Livro Branco da Ciência, Tecnologia e Inovação, Brasília, DF, CNPq/MCT, 2002.

Morgan, G. Paradigms, Metaphors, and Puzzle Solving in Organization Theory. Adm. Science Quarterly, v 25, 1980, p. 605-622

cross ${ }^{\text {ref }}$

Nelson, R. National innovation systems: a comparative analysis. Oxford: Oxford University, 1993.

Nicolsky, R. "Inovação tecnológica industrial e desenvolvimento sustentado", Parcerias Estratégicas. 13, 2001, p. 80108.

Organização para a Cooperação Econômica e o Desenvolvimento - OCDE. Main Science and Technology Indicators, 2 , Paris, OCDE, 1997a.

OCDE. Diffusing technology to industry: government policies and programmers, Working Paper, vol. V, 33, Paris, OCDE, $1997 b$.

OCDE. Industrial competitiveness, Paris, OCDE, 1996.

OCDE. Managing national innovation systems, Paris, OCDE, 1999.

OCDE. Brazil - Economic survey, Paris, OCDE, 2001.

OCDE. Medição de atividades científicas e tecnológicas. Paris, OCDE, 1979.

Manual Frascati. Brasília: CNPq, 2001.

Pavitt, K. The social shape of the national science base. Research Policy, v. 27, n.8. 1998, p. 793-805.

cross ${ }^{\text {ref }}$

What makes basic research economically useful? Research Policy, v. 20, n.2, 1991, p. 109-119. 
Porter, M. E. The competitive advantage of nations, New York, Macmillan, 1990.

PNUD. Human Development Report 2003: Millennium Development Goals: A compact among nations to end human poverty, New York, Oxford University Press for de UNDP, 2003.

Informes sobre el desarrollo humano, Brasília, DF: PNUD-IPEA, 2002.

Roussel, P. A; Saad, K. N, e, Bohlin, N. Pesquisa \& Desenvolvimento, São Paulo, SP, Makron Books, 1992.

Schwartzman, S. Um espaço para a Ciência: A formação da comunidade cientifica no Brasil, Brasília, DF, CNPq/MCT. 2001, 357 págs.

(coord.). Ciência e tecnologia no Brasil: Uma nova política para um mundo global, 3 vols., Rio de Janeiro, RJ, Editora Fundação Getúlio Vargas, 1995.

, Krieger, E. et. al. "Ciência e tecnologia no Brasil: Uma nova política para um mundo global”, in Simon Schwartzman (coord.), Ciência e tecnologia no Brasil: Política industrial, mercado de trabalho e instituições de apoio, vol. 2, Rio de Janeiro, RJ, Editora da Fundação Getúlio Vargas, 1995, p. 1-59.

Simon, H. Rationality as process and as product of thought. American Economic Review, v. 68, n. 2, 1978, p.1-16.

Shin, T., et. al. The first survey for Science and Technology Forecasting; Korea's Future Technology, Seoul; Science and Technology Policy Institute (STEPI), 1994.

Science and Technology Policy Institute. A Long-range Plan for Science and Technology toward the Year 2010, Seoul, Science and Technology Policy Institute (STEPI), 1995.

\section{Dados completos do primeiro autor:}

Nome completo: José Matias-Pereira

Filiação institucional: Universidade de Brasília

Departamento: Programa de Pós-Graduação em Administração

Função ou cargo ocupado: Professor e coordenador-adjunto

Endereço completo para correspondência (bairro, cidade, estado, país e CEP): SHIS QI 26 Conj. 5, casa

22, Lago Sul, Brasília - DF, 71670-050.

e-mail:matias@unb.br; matias.pereira@brturbo.com.br 